\title{
Percepción de Estrés Escolar y Ritmo Circadiano de Cortisol en el Síndrome de Turner
}

\author{
Marcela López \\ Centro de Investigación en Procesos Básicos, Metodología y Educación de la Universidad \\ Nacional de Mar del Plata, Mar del Plata, Província de Buenos Aires, Argentina \\ María José Aguilar ${ }^{1}$ \\ Consejo Nacional de Investigaciones Cientificas y Técnicas, Buenos Aires, \\ Província de Buenos Aires, Argentina \\ Centro de Investigación en Procesos Básicos, Metodología y Educación de la Universidad \\ Nacional de Mar del Plata, Mar del Plata, Província de Buenos Aires, Argentina \\ Verónica Zabaletta \\ Centro de Investigación en Procesos Básicos, Metodología y Educación de la Universidad \\ Nacional de Mar del Plata, Mar del Plata, Província de Buenos Aires, Argentina
}

\section{Resumen}

El Síndrome de Turner corresponde a uno de los trastornos cromosómicos, no heredables, con mayor incidencia poblacional. Las particularidades físicas, neuropsicológicas y psicosociales, relacionadas con la expresión del síndrome, posicionan a las mujeres con este diagnóstico como una población potencialmente vulnerable al estrés. El objetivo del trabajo fue analizar comparativamente dos medidas de estrés en niñas y adolescentes con diagnóstico de Síndrome de Turner, la percepción de estrés a través del Cuestionario para el estrés escolar y la pendiente circadiana de cortisol por determinación de cortisol salival. El análisis de los datos del cuestionario dio cuenta de valores de estrés escolar medios y bajos. Sin embargo, en la totalidad de las participantes se observó alteración de la pendiente de cortisol con valores vespertinos altos, independiente de la etapa de desarrollo puberal, del cariotipo y de los tratamientos hormonales que recibían al momento del estudio. Estos resultados, si bien limitados debido al tamaño de la muestra, podrían relacionarse con la morfología cerebral atípica reportada para esta población en ciertas estructuras corticales y subcorticales. Asimismo, las diferencias encontradas entre las medidas objetiva y subjetiva de estrés podrían deberse a que los altos niveles de cortisol generan dificultades en procesos cognitivos por lo que la percepción de las señales de alerta se encontrarían disminuidas o anuladas en esta población, restándoles la posibilidad de responder a estímulos potencialmente peligrosos.

Palabras clave: Síndrome de Turner, estrés escolar, pendiente circadiana de cortisol

\section{Percepção de Estresse Escolar e Ritmo Circadiano de Cortisol na Sindrome de Turner}

\section{Resumo}

A Síndrome de Turner é um dos transtornos cromossômicos, não herdáveis, com maior incidência na população. As particularidades físicas, neuropsicológicas e psicossociais, relacionadas com a expres-

Endereço para correspondência: Facultad de Psicología, Centro de Investigación en Procesos Básicos, Metodología y Educación, Universidad Nacional de Mar del Plata, Complejo Universitario, Funes 3250, Cuerpo V, Nivel III, Mar del Plata, Argentina 7600. E-mail: mclopez@mdp.edu.ar, majoaguilar1@gmail.com e vzabaletta@gmail.com 
são da síndrome, posicionam às mulheres com este diagnóstico como uma população potencialmente vulnerável ao estresse. $\mathrm{O}$ objetivo do trabalho foi analisar comparativamente duas medidas de estresse em crianças e adolescentes com diagnóstico de Síndrome de Turner, a percepção de estresse através do Questionário para o estresse escolar e a pendente circadiana de cortisol para determinação de cortisol salival. A análise dos dados do questionário mostrou valores de estresse escolar médios e baixos. Não obstante na totalidade das participantes observou-se uma alteração da pendente de cortisol com valores vespertinos altos, com independência da etapa de desenvolvimento puberal, do cariótipo e dos tratamentos hormonais que elas recebiam ao momento da pesquisa. Estes resultados, conquanto limitados devido ao tamanho da amostra, poderiam relacionar-se com a morfologia cerebral atípica reportada para esta população em certas estruturas corticais e subcorticais. Assim mesmo a diferença encontrada entre as medidas objetiva e subjetiva de estresse poderiam ser causa de que os altos níveis de cortisol geram dificuldades em processos cognitivos pelo que a percepção dos sinais de alerta estão diminuídas ou anuladas nesta população, não tendo a possibilidade de responder aos estímulos potencialmente perigosos.

Palavras-chave: Síndrome de Turner, estresse escolar, pendente circadiana de cortisol.

\title{
Scholar Stress Perceptions and Cortisol Circadian Rhythm in Turner's Syndrome
}

\begin{abstract}
Turner's syndrome corresponds to one of the chromosomal disorders, not inheritable, with the highest population incidence. The physical, neuropsychological and psychosocial particularities, related to the expression of the syndrome, position women with this diagnosis as a population potentially vulnerable to stress. The aim of the work was to analyze comparatively two measures of stress in girls and teenagers with diagnosis of Turner's syndrome, their stress perception across the Questionnaire for school stress and the circadian cortisol slope determined by salivary cortisol. The analysis of the information from the questionnaire shows average and low levels of school stress. However, an alteration of cortisol slope with high evening values was observed in every participant, independent from the stage of pubertal development, their karyotype and the hormonal treatment they have received at the time of the study. These results, although limited by the size of the sample, could be related to the atypical cerebral morphology reported for this population in certain cortical and subcortical structures. Also, the differences founded between objective and subjective measures of stress could be due to high levels of cortisol that generate difficulties at cognitive processes so that the perception of the signs of alert would be diminished or annulled in this population, reducing their possibility to respond to potentially dangerous stimuli.
\end{abstract}

Keywords: Turner's syndrome, school stress, circadian cortisol slope.

En la actualidad existe consenso acerca de la importancia de realizar investigaciones que integren variables psicológicas y biológicas, dando lugar a trabajos interdisciplinarios donde convergen diferentes disciplinas como la psicología, la neuropsicología y la biología, entre otras. En este contexto, el estudio de las características psicológicas y fisiológicas de personas con trastornos genéticos abre nuevas líneas de investigación destinadas a diseñar modelos explicativos que permitan analizar la interacción de factores psicológicos, biológicos y ambientales en el desarrollo (Plomin, De Fries, McClearn, \& Guffin, 2002).

El Síndrome de Turner (ST) es un trastorno genético, determinado por la deleción total o parcial del cromosoma $\mathrm{X}$ en el sexo femenino. Corresponde a uno de los trastornos cromosómicos no heredables con mayor incidencia poblacional, 1:1900 niñas nacidas vivas (Murphy, Mazzocco, Gerner, \& Henry, 2006). Las características físicas más frecuentes en mujeres con 
este diagnóstico comprenden talla baja $(100 \%$ de los casos), ausencia de maduración puberal (96\%) y esterilidad (99\%; Ramos, 2003). En relación al perfil cognitivo, diversas investigaciones consideran que las habilidades verbales y las capacidades intelectuales permanecen conservadas (Doswell, Visootsak, Brady, \& Graham, 2006; Kesler, Garrett, et al., 2004); sin embargo, se han reportado déficits selectivos de ciertos dominios como habilidades visuoespaciales que incluyen procesos de percepción espacial, integración visual-motora y orientación izquierda-derecha, así como dificultades en tareas de atención que requieren control de la impulsividad y automonitoreo (Mazzocco, 2006; Ross, Roeltgen, \& Zinn, 2006; Schmidt, Rubinow, \& Bondy, 2006; Zinn et al., 2007). Asimismo, suelen presentar problemas en la memoria no verbal, especialmente en la memoria de trabajo $y$, dificultades en el manejo del factor numérico (matemática y cálculos), en la planificación y ejecución de tareas complejas y en la comprensión y generación de conceptos abstractos (Mazzocco, 2001). Lo expuesto trae aparejado problemas en el aprendizaje, concretamente en procesamiento de ideas, relaciones visión-espacio y discriminación izquierda derecha.

Otra característica ampliamente descripta en la literatura es la dificultad de las mujeres con diagnóstico de ST para interactuar con sus pares, asociada a una baja autoestima, inmadurez emocional y ansiedad social (Danielewicz \& Pisula, 2005; Mazzocco, 2006; Suzigan et al., 2004). A este respecto Sutton et al. (2005) expresa que la existencia de un estigma social asociado a la baja estatura es percibido por las niñas y adolescentes con diagnóstico de ST como una fuente de estrés.

Se puede considerar que las características detalladas anteriormente podrían posicionar a las mujeres con este diagnóstico como una población potencialmente vulnerable al estrés. En este punto si se tiene en cuenta que la muestra analizada corresponde a niñas y adolescentes, dentro de los múltiples contextos en los que se desenvuelven a lo largo del desarrollo, el contexto escolar se constituye en un ámbito que por sus características y exigencias puede ser un factor potencial de estrés. El inicio de la escolarización y su posterior desarrollo, sin olvidar el tránsito de un nivel educativo a otro, suelen ser eventos estresantes para los niños. Martínez-Díaz y DíazGómez (2007) definen el estrés escolar como el malestar que un estudiante puede presentar a raíz de factores físicos, emocionales, ambientales o interrelacionales que pueden influir significativamente, tales como presión sobre las competencias individuales para afrontar el contexto escolar. El modelo transaccional (Barraza-Macías, 2006) explica el desarrollo de estrés como un proceso dinámico de adaptación-desadaptación, mediatizado por la evaluación que la persona realiza de la situación y de sus propias capacidades para afrontarla. Este modelo introduce la mediación cognitiva como pieza fundamental en el desarrollo de estrés, es un modelo abarcativo en tanto permite el acercamiento a las dimensiones biológica, psicológica y socio-culturales sin que se excluyan mutuamente, considerando que no es el suceso medioambiental, ni la respuesta de la persona lo que define al estrés, sino la percepción que tiene el individuo de la situación psicológica (Lazarus \& Folkman, 1986).

Existen diversas medidas para valorar el grado de estrés que experimenta una persona, por un lado, la percepción que tiene de las situaciones estresantes es un indicador subjetivo válido, por otro lado, desde una perspectiva biológica, un indicador objetivo lo constituyen las concentraciones diarias de cortisol; considerándose que en situaciones de estrés los niveles de esta hormona aumentan, alterándose el ritmo circadiano de cortisol. Según este ritmo, los niveles de cortisol comienzan a incrementarse en la última hora de sueño, alcanzando su punto más alto aproximadamente treinta minutos después de despertar, descendiendo rápidamente durante los siguientes treinta a sesenta minutos, para continuar su declinación de forma paulatina a lo largo del resto del día, alcanzando sus niveles más bajos al final de la fase de actividad diurna. El procesamiento individual ante los estímulos está íntimamente relacionado con estas trazas biológicas, lo que da como resultado los diferentes patrones de afrontamiento al estrés, siendo el fenotipo vulnerable aquel que presenta hiperac- 
tividad del eje hipotálamo-hipófiso-adrenal, con altos niveles de cortisol diarios (López Mato, 2004).

De acuerdo a lo expuesto el objetivo del trabajo fue analizar comparativamente las dos medidas de estrés en niñas y adolescentes con diagnóstico de ST, su percepción a través de un cuestionario que valora estrés escolar, y la pendiente circadiana de cortisol a través a determinación de cortisol salival.

\section{Métodos}

\section{Diseño}

Se trabajó con un diseño de tipo ex post facto retrospectivo con un grupo simple según la clasificación de Montero y León (2007).

\section{Participantes}

La muestra fue intencional y corresponde a niñas y adolescentes con diagnóstico de Síndrome de Turner $(N=6)$, con edades entre 7 y 16 años. Las participantes fueron contactadas mediante convenios específicos con los centros de salud referentes en la atención del síndrome de la ciudad de Mar del Plata. Se excluyeron niñas y/o adolescentes con un índice de masa corporal mayor de $35 \mathrm{~kg} / \mathrm{m}^{2}$, diabetes, insuficiencia hepática o renal crónica, tratamiento con corticoides en el último año y niñas/adolescentes que hayan cursado alguna enfermedad aguda en las últimas dos semanas.

Todas las familias abordadas presentan un nivel socioeconómico medio, realizando las niñas y adolescentes escolaridad regular en instituciones educativas públicas y privadas de la ciudad de Mar del Plata. La totalidad de la muestra ha recibido hormona de crecimiento desde una edad temprana, lo cual les permitió a las adolescentes alcanzar percentiles de estatura dentro de un rango normal. Tres de las participantes han alcanzado la pubertad, dos de forma espontánea y una con tratamiento estrogénico. Cabe considerar que ambos tratamientos hormonales son considerados fundamentales para optimizar la calidad de vida de esta población, debido a su impacto en el desarrollo psicosocial.

En la Tabla 1 se presenta, para cada participante, una descripción de los parámetros biológicos más significativos en el desarrollo.

Tabla 1

Información Clínica de las Niñas y Adolescentes con Diagnostico de ST (N=6)

\begin{tabular}{|c|c|c|c|c|c|c|}
\hline Participantes & 1 & 2 & 3 & 4 & 5 & 6 \\
\hline Cariotipo & $45, \mathrm{X} 0$ & $\begin{array}{c}45, \mathrm{X} 0 / \\
46, \mathrm{XXdelq} 10 *\end{array}$ & $\begin{array}{c}45, \mathrm{X} 0 / \\
46, X X d e l p 21 *\end{array}$ & $\begin{array}{c}46, \mathrm{XX} / 45, \mathrm{X} 0 / \\
47, \mathrm{XXX}^{*}\end{array}$ & $\begin{array}{l}46, \mathrm{XX} / \\
45, \mathrm{X} 0 *\end{array}$ & $45, \mathrm{X} 0$ \\
\hline Edad actual & 7 años & 7 años & 13 años & 14 años & 15 años & 16 años \\
\hline $\begin{array}{l}\text { Tratamientos } \\
\text { hormonales }\end{array}$ & H. C $* *$ & H. C & H. C & -------- & -------- & estrógeno \\
\hline Pubertad & $\begin{array}{c}\sin \\
\text { pubertad }\end{array}$ & sin pubertad & sin pubertad & $\begin{array}{l}\text { con pubertad } \\
\text { espontanea }\end{array}$ & $\begin{array}{c}\text { con } \\
\text { pubertad } \\
\text { espontanea }\end{array}$ & $\begin{array}{c}\text { con } \\
\text { pubertad } \\
\text { inducida }\end{array}$ \\
\hline
\end{tabular}

Nota. ${ }^{*}$ Cariotipo mosaico; ** Hormona de crecimiento.

\section{Materiales}

Para evaluar la percepción de estrés escolar, se utilizó el test: Cuestionario para el estrés escolar (QSS; Bringhenti, 1996), compuesto por 35 ítems sobre las variables del estrés y sus indicadores, con un formato valorativo de escala tipo Likert. Esta escala se puntúa con un gra- diente entre 1 y 4 , siendo la puntuación mínima 1 (correspondiente con no verdadero) y máxima 4 (correspondiente con verdadero). El cuestionario consta de tres sub-escalas. La primera conformada por 20 ítems, contempla factores emotivos- motivacionales, que evalúa aspectos de insatisfacción en relación con los docentes, 
manifestaciones de agotamiento emocional y decepción causadas por la evolución de los resultados obtenidos en la escuela. Estima el rol de la relación maestro-estudiante en la caracterización de la vida escolar de los niños. La segunda sub-escala, que consta de ocho ítems, mide la tensión interpersonal y está destinada a recoger las sensaciones de fastidio e impresiones de hostilidad por parte de algunos integrantes del grupo de la clase, comportamientos que aumentan la sensación de cansancio emotivo y la falta de tolerancia recíproca. La tercera sub-escala integrada por siete ítems, evalúa aspectos sociales de autoestima y adaptación con independencia de los resultados escolares. Está relacionada con la aceptación y consideración de parte de compañeros y maestros que podrían salvaguardar condiciones mínimas de adaptación a la vida escolar en el caso que los problemas de relación y de rendimiento fueran limitados.

Paralelamente, para caracterizar la pendiente circadiana de cortisol, se determinó cortisol libre en saliva, el cual ofrece ventajas sobre otras mediciones como suero u orina. El método de recolección es indoloro y no invasivo, reduciendo el estrés que produce la extracción y evitando el sesgo que pudiera producirse a raíz del procedimiento. Se ha comprobado que las cifras de cortisol salival representan adecuadamente a las cifras plasmáticas, siendo una medida que se mantiene estable por varias semanas y permite cuantificar de forma exacta y eficiente el cortisol biológicamente activo (Mirasoli, Deo, Lewis, Roda, \& Daunert, 2002).

El cortisol salival se midió por radioinmunoanálisis competitivo, utilizando el kit comercial Coat-a-Count con estándares diluidos 1/10. El estudio se realizó en el Laboratorio de Análisis Clínico y Endocrinológico Laycet (90240) de Mar del Plata (Argentina); acreditado por la Fundación Bioquímica Argentina con evaluación de calidad y permiso para el uso de trazadores radiactivos in vitro en humanos. Para analizar la curva circadiana de cortisol se utilizó el protocolo de Adam y Kumari (2009), estos autores proponen la obtención de un coeficiente que refleja la declinación diaria del cortisol. El mismo se obtiene restándole al valor vespertino de cortisol (18hs.) el valor matutino ( $8 \mathrm{hss}$.) y dividiendo el número resultante por la cantidad de horas que separan ambas muestras.

\section{Procedimiento}

En una primera etapa se estableció contacto con los centros de salud y los profesionales que atienden niñas y adolescentes con diagnóstico de ST (consultorios endocrinológicos). A través de estos últimos, se contactó a las familias y se les explicó el objetivo de la investigación y las características de la misma, solicitándoles la firma del consentimiento informado y del asentimiento. Asimismo se les solicitó el permiso para contactar las instituciones educativas a la que asisten sus hijas. Posteriormente se administró el instrumento de evaluación de estrés escolar en entrevistas personales con las niñas y adolescentes.

Paralelamente a cada familia se les explicó el protocolo de extracción de la muestra salival, indicándoles no cepillarse los dientes antes de recolectar la muestra para evitar el sangrado de las encías, no ingerir agua o alimentos, ni utilizar inhaladores que contengan esteroides. Se obtuvo aproximadamente $1 \mathrm{ml}$ de saliva, recolectada por salivación directa dentro de un tubo de vidrio estéril sin aditivos, que se almacenó a $4^{\circ} \mathrm{C}$. Se realizaron dos tomas diarias, inmediatamente después de levantarse (matutino: 8:00) y a media tarde (vespertino: 18:00 horas). Para neutralizar el efecto de posibles demandas ambientales, se obtuvieron cuatro muestras de saliva de cada participante, dos de las mismas corresponden a una jornada escolar y las otras a una jornada de descanso, tomando ésta última como situación basal (Moya-Albiol, Serrano, González-Bono, Rodríguez-Alarcón, \& Salvador, 2005).

Para el análisis de los datos se utilizó el Statistical Package for the Social Sciences (SPSS 11.5), aplicando pruebas estadísticas no paramétricas debido al tamaño y distribución de la muestra. Se consideró significativo un valor $=<$ .05. Los valores medios obtenidos fueron comparados con un estudio similar realizado con niñas y adolescentes sin diagnóstico de ST. 


\section{Consideraciones Éticas}

La participación fue voluntaria y sujeta al consentimiento informado de los padres y al asentimiento de las participantes. Durante el desarrollo del trabajo se respetaron los principios éticos de la investigación con seres humanos, procurándose las condiciones necesarias para proteger la confidencialidad y actuar en beneficio de los participantes.

\section{Resultados}

En la Tabla 2 se presenta el análisis descriptivo y de diferencia de medias para la variable estrés escolar y sus dimensiones en niñas y adolescentes con diagnóstico de ST comparadas con un grupo de referencia evaluado en una investigación previa realizada por los autores (Zabaletta, Gasparri, Gorostegui, Aguilar, \& López, 2011).

Tabla 2

Medidas de Tendencia Central, Desvío Estándar y Prueba U de Mann-Whitney sobre la Variable Estrés Escolar y sus Dimensiones

\begin{tabular}{cccccc}
\hline Grupo & $\begin{array}{c}\text { QSSEM } \\
\text { (Emotivo- } \\
\text { Motivacional) }\end{array}$ & $\begin{array}{c}\text { QSSTI } \\
\text { (Tensión } \\
\text { interpersonal) }\end{array}$ & $\begin{array}{c}\text { QSSAA } \\
\text { (Autoestima- } \\
\text { Adaptación escolar) }\end{array}$ & $\begin{array}{c}\text { QSS } \\
\text { Estrés escolar }\end{array}$ \\
\hline 1 & $\bar{X}(S D)$ & $38.17(9.32)$ & $17.33(3.26)$ & $15.17(4.35)$ & $70.67(8.93)$ \\
$2^{*}$ & $\bar{X}(S D)$ & $37.17(4.87)$ & $14.83(5.41)$ & $12.33(2.80)$ & $64.33(11.09)$ \\
$\begin{array}{c}U \text { de } \\
\text { Mann-Whitney }\end{array}$ & Sig. & .378 & .936 & .191 & .227 \\
\hline
\end{tabular}

Notas. Grupo 1= niñas y adolescentes con diagnóstico de Síndrome de Turner. Grupo 2= niñas y adolescentes sin diagnóstico de Síndrome de Turner. *(Zabaletta et al., 2011).

Las niñas y adolescentes con diagnóstico de ST presentan valores superiores en estrés escolar y sus dimensiones, en relación a su grupo de referencia. Sin embargo estas diferencias no son significativas ya que superan el nivel crítico de .05 .
En la Tabla 3 se presentan las medidas de tendencia central y variabilidad de los valores de cortisol salival matutinos (8hs.) y vespertinos (18hs.). La Tabla 4 compara ambas jornadas a través de la prueba de Wilcoxon.

Tabla 3

Cortisol Salival (ug/dl) Matutino y Vespertino para Ambas Jornadas

\begin{tabular}{|c|c|c|c|c|}
\hline & \multicolumn{2}{|c|}{ Jornada escolar } & \multicolumn{2}{|c|}{ Jornada de descanso } \\
\hline & $\begin{array}{c}\text { Cortisol salival } \\
\text { (8hs.). }\end{array}$ & $\begin{array}{c}\text { Cortisol salival } \\
\text { (18hs.). }\end{array}$ & $\begin{array}{c}\text { Cortisol salival } \\
\text { (8hs.). }\end{array}$ & $\begin{array}{c}\text { Cortisol salival } \\
\text { (18hs.). }\end{array}$ \\
\hline$(S D)$ & $.9393(.1814)$ & $.7367(.0896)$ & $.8117(.0898)$ & $.7017(.0985)$ \\
\hline
\end{tabular}

Tabla 4

Prueba de Wilcoxon de Comparación de Grupos: Jornada Escolar y Jornada de Descanso

Cortisol salival (8hs.). Jornada escolar - Jornada descanso
Cortisol salival (18hs.). Jornada escolar - Jornada descanso 
Los resultados presentados no arrojan diferencias significativas entre los valores de cortisol matutinos y vespertinos para ambas jornadas. La prueba de Wilcoxon muestra un nivel de significatividad superior a .05 no hallándose diferencias en la pendiente de cortisol en ambas jornadas.
En las Figuras 1 y 2 se presentan las curvas de descenso de cortisol comparando valores observados y esperados para ambas jornadas. Se considera para el valor esperado una estimación de descenso de la curva de cortisol del 50\% entre valores matutinos y vespertinos (Lépez, Caamaño, Romero, Fiedler, \& Araya, 2010).

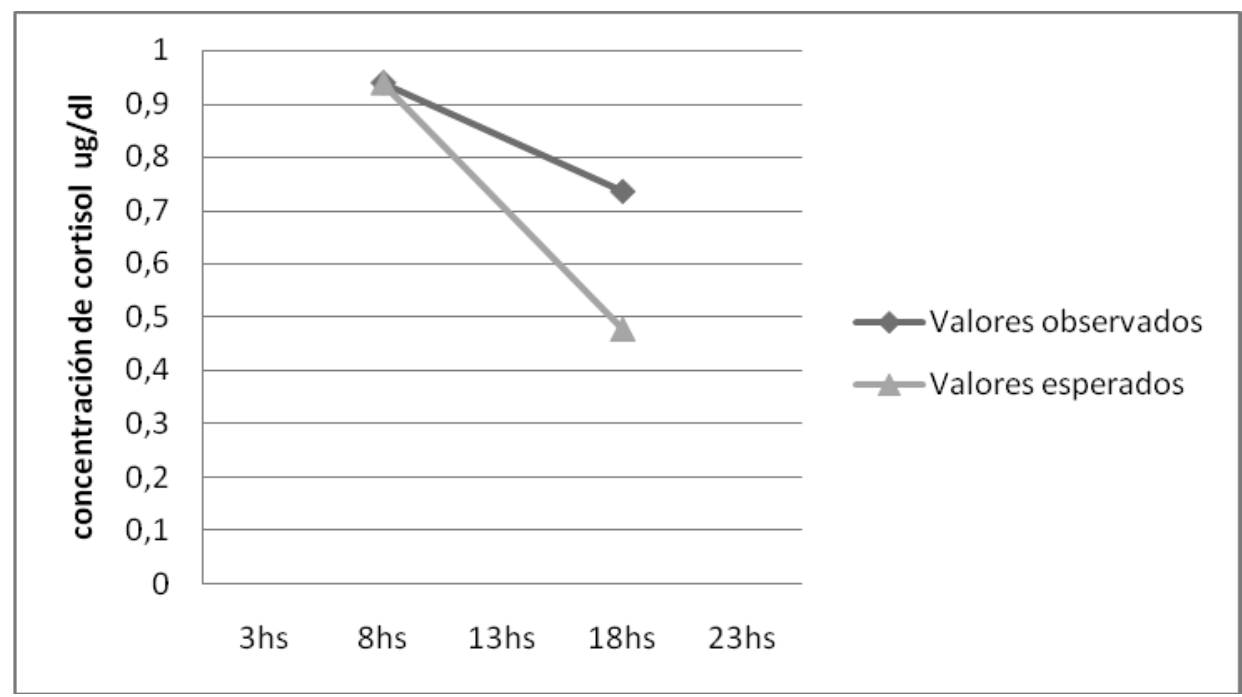

Figura 1. Descenso de cortisol jornada escolar.

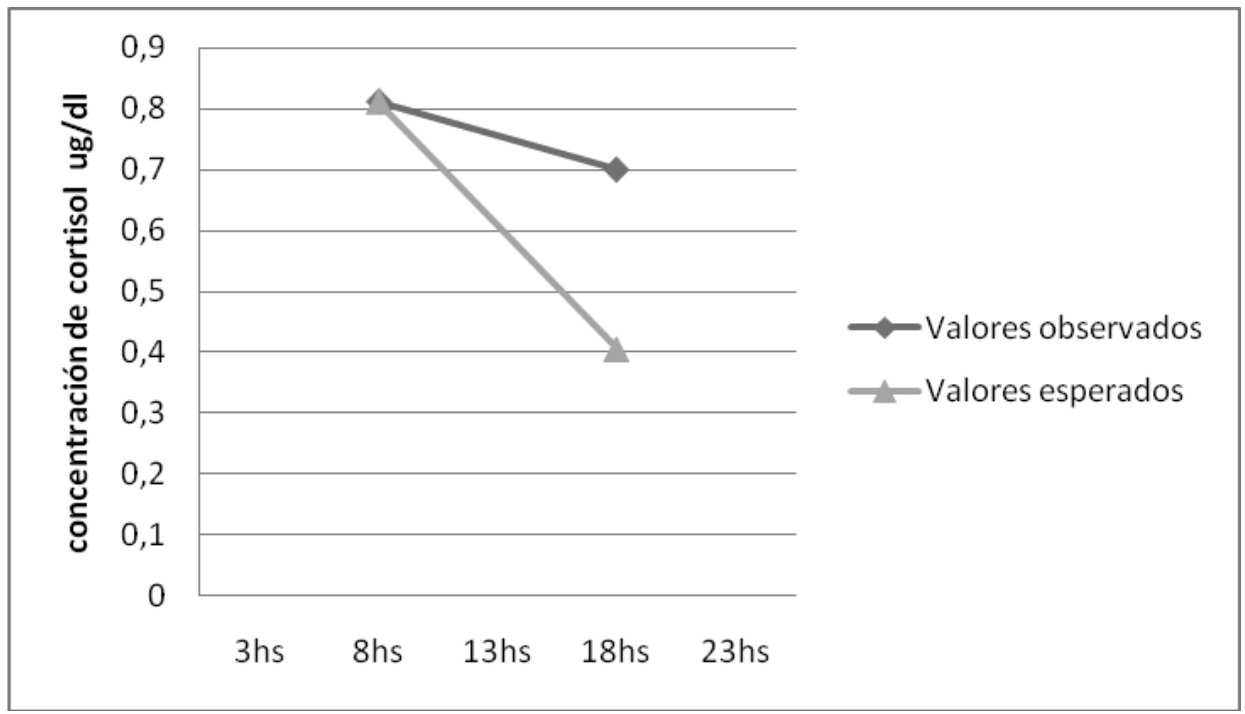

Figura 2. Descenso de cortisol jornada de descanso.

El nivel de cortisol vespertino observado para ambas jornadas (.736 y .701) es mayor al esperado (.477 y .405), dando cuenta de un menor descenso diario de la concentración cortisol, con altos valores vespertinos.

En la Tabla 5 se muestran las medidas de tendencia central, variabilidad y comparación de los coeficientes observados y esperados considerando conjuntamente la jornada escolar y de descanso.

La prueba Wilcoxon arroja un nivel de significatividad menor a .05 rechazándose la hipótesis de igualdad de medias. Indicando alteración del descenso circadiano de cortisol en el total de 
Tabla 5

Coeficientes de Declinación de la Curva Circadiana de Cortisol y la Prueba de Wilcoxon de Comparación de Grupos

\begin{tabular}{ccc}
\hline & Coeficientes observados & Coeficientes esperados \\
\hline $\bar{X}(S D)$ & $-.0217(.0244)$ & $-.0470(.0089)$ \\
Sig. Bilateral entre ambos coeficientes. & .028 & \\
\hline
\end{tabular}

las participantes para ambas jornadas, no hallándose diferencias significativas entre las niñas sin pubertad y las adolescentes o púberes (jornada escolar $p=.426$; jornada de descanso $p=.341$ ).

\section{Discusión}

El análisis de los resultados da cuenta que las niñas y adolescentes con diagnóstico de ST presentan una percepción de estrés escolar con valores medios y bajos; sin embargo, en la totalidad de las participantes se observó alteración de la curva circadiana de cortisol, con altos niveles de cortisol vespertinos en las dos jornadas evaluadas (escolar y de descanso).

Estos resultados, si bien limitados debido al tamaño de la muestra, podrían relacionarse con la morfología cerebral reportada para esta población. Los principales hallazgos dentro de esta línea revelan la presencia de una organización cortical atípica, especialmente en el hemisferio cerebral derecho con déficits en el funcionamiento de los circuitos frontotemporales (Tamm, Menon, Allan, \& Reiss, 2003). A través del análisis de imágenes de resonancia magnética se ha observado una disminución en el volumen de materia gris de los lóbulos occipital y temporal derechos y frontal izquierdo y derecho (Brown et al., 2004; Danielewicz \& Pisula, 2005; Doswell et al., 2006; Hart, Davenport, Hooper, \& Belger, 2006; Kesler, Garrett, et al., 2004; Ross et al., 2006). Asimismo se han reportado alteraciones en estructuras subcorticales como cerebelo, protuberancia, tálamo, amígdala e hipocampo.

Kesler, Haberecht, et al. (2004) y McCauley y Sybert (2006) han observado un agrandamiento en el volumen amigdalino izquierdo y una reducción del hipocampo derecho. Ambas estructuras son claves en la regulación del eje hipotálamo-hipofiso-adrenal (HPA). El hipocampo es una de las dianas de la hormona cortisol, posee en su estructura las mayores concentraciones de receptores para corticosteroides del cerebro de los mamíferos. Una de sus funciones neuroendocrinas es regular la cantidad de cortisol circundante a través de procesos de retroalimentación negativa, inhibiendo el eje HPA. Por su parte, la amígdala secreta un neurotransmisor activador del eje (hormona liberadora de corticotrofina hipotalámica). Las atrofia hipocampal y la hipertrofia amigdalina podrían inducir una hiperactividad de este eje lo que conlleva a la hipercortisolemia permanente con una alteración circadiana del ritmo de cortisol.

En este punto es importante tener en cuenta que el cortisol es considerado la hormona del estrés en humanos y que experiencias estresantes tempranas crónicas y continuas en la vida, juegan un rol modulador importante sobre el sistema fisiológico del estrés. Maldonado et al., (2008) refieren que la secreción de cortisol es mayor frente a acontecimientos vitales que frente a acontecimientos aislados por muy estresantes que éstos se perciban. A este respecto, las mujeres con diagnóstico de $\mathrm{ST}$ atraviesan durante su desarrollo diversas situaciones disruptivas susceptibles de generar estrés, consecuencia de las características físicas y psicológicas particulares de la expresión del síndrome.

Asimismo, los altos niveles de cortisol generan dificultades en procesos cognitivos (Murphy et al., 2006), hecho que podría explicar las diferencias encontradas entre las medidas objetiva y subjetiva de estrés; considerando que una alta reactividad del eje biológico podría suprimir la percepción de la respuesta emocional, por lo 
que la percepción de las señales de alerta se encontraría disminuida o anulada, restándoles a las niñas y adolescentes con diagnóstico de ST la posibilidad de responder a estímulos potencialmente peligrosos, capacidad necesaria para una mejor adaptación.

Esta disfuncionalidad en la pendiente diaria de cortisol podría ser considerada una característica propia de la expresión del ST ya que se evidenció en el total de las participantes, independiente de la etapa de desarrollo puberal (premenárquicas y pos-menárquicas), del cariotipo (línea pura y mosaicos) y de los tratamientos hormonales que recibían al momento del estudio (hormona de crecimiento y hormona estrogénica). Por otro lado, es importante destacar que no se hallaron diferencias significativas entre las jornadas escolares y de descanso, dato que podría estar minimizando el impacto de factores ambientales y fortaleciendo la hipótesis que considera a la alteración en la curva de descenso diario de cortisol como una característica propia de la expresión del síndrome.

En un futuro sería interesante contrastar estos resultados a través de un estudio caso-control para afirmar que los altos niveles de cortisol vespertinos son patognomónicos de la población con diagnóstico de ST, considerando que a este respecto existen reportes de estudios con resultados disímiles, mientras Gravholt (2004) refiere un funcionamiento normal del eje adrenal en mujeres con diagnóstico de ST, un estudio reciente realizado por Onder et al. (2012) reportó altos valores de cortisol en mujeres con este diagnóstico.

Asimismo ahondar en la relación entre morfología cerebral atípica y pendiente diurna de cortisol a través de un estudio simultáneo y comparativo que incluya el uso de imágenes de resonancia magnética permitiría contrastar los supuestos expuestos y dar cuenta de la alta sensibilidad biológica al contexto de las mujeres con diagnóstico de ST, ya que una activación persistente del eje HPA está asociada con sesgos emocionales y cognitivos que impactan en el desarrollo de procesos fundamentales para el aprendizaje.

\section{Referencias}

Adam, E. K., \& Kumari, M. (2009). Assessing salivary cortisol in large-scale, epidemiological research. Psychoneuroendocrinology, 34, 14231436.

Barraza-Macías, A. (2006). Un modelo conceptual para el estudio del estrés académico. Recuperado el 28 de diciembre, 2010, en http:// www.psicologiacientifica.com/bv/psicologia167-1-un-modelo-conceptual-para-el-estudiodel-estres-academico.html

Bringhenti, F. (1996). Lo stress scolástico e la sua valutazione. Psicologia e Scuola, 81, 3-13.

Brown, W., Kesler, S., Eliez, S., Warsofsky, I., Haberecht, M., \& Reiss, A. (2004). A volumetric study of parietal lobe subregions in Turner Syndrome. Developmental Medicine \& Child Neurology, 46(9), 607-609.

Danielewicz, D., \& Pisula, E. (2005). Self-esteem evaluation of girls of Turner Syndrome. Annales Universitatis Mariae Curie-Skłodowska LublinPolonia, Sectio D, Medicina, 60(4, Suppl. 16), 329-332.

Doswell, B. H., Visootsak, J., Brady, A. N., \& Graham, J. M. (2006). Turner Syndrome: An update and review for the primary pediatrician. Clinical Pediatrics, 45(4), 301-313.

Gravholt, C. H. (2004). Epidemiological, endocrine and metabolic features in Turner Syndrome. European Journal of Endocrinology, 151, 657-687.

Hart, S., Davenport, M., Hooper, S., \& Belger, A. (2006). Visuospatial executive function in Turner Syndrome: Functional MRI and neurocognitive findings. Brain, 129(5), 1125-1136.

Kesler, S., Garrett, A., Bender, B., Yankowitz, J., Zeng, S. M., \& Reiss, A. (2004). Amygdala and hippocampal volumes in Turner Syndrome: A high-resolution MRI study of X-monosomy. Neuropsychologia, 42, 1971-1978.

Kesler, S., Haberecht, M., Menon, V., Warsofsky, I., Dyer-Friedman, J., Neely, J., \& Reiss, A. (2004). Functional neuroanatomy of spatial orientation processing in Turner Síndrome. Cerebral Cortex, 14(2), 174-180.

Lazarus, R. S., \& Folkman, S. (1986). Estrés y procesos cognitivos. Barcelona, España: Martínez Roca. 
Lépez, M., Caamaño, E., Romero, C., Fiedler, J., \& Araya, V. (2010). Determinación de los niveles de cortisol salival en una muestra de sujetos de Santiago de Chile. Revista Médica de Chile, 138(2), 1-7.

López Mato, A. M. (2004). Psiconeuroinmunoendocrinología II. Buenos Aires, Argentina: Polemos.

Maldonado, E. F., Fernandez, F. J., Trianes, M. V., Wesnes, K., Petrini, O., Zangara, A., ...Ambrosetti, L. (2008). Cognitive performance and morning levels of salivary cortisol and amylase in children reporting high vs. low daily stress perception. The Spanish Journal of Psychology, 11(1), 3-15.

Martínez-Díaz, E. S., \& Díaz-Gómez, D. A. (2007). Una aproximación psicosocial al estrés escolar. Educación y Educadores, 10(2), 11-22.

Mazzocco, M. (2001). Math learning disability and math LD subtypes: Evidence from studies of Turner syndrome, fragile X syndrome and neurofibromatosis type 1. Journal of Learning Disabilities, 34(6), 520-533.

Mazzocco, M. (2006). The cognitive phenotype of Turner Syndrome: Specific learning disabilities. International Congress Series 1298, 83-92.

McCauley, E., \& Sybert, V. (2006). Social and behavioral development of girls and women with Turner Syndrome. International Congress Series, 1298, 93-99.

Mirasoli, M., Deo, S. K., Lewis, J. C., Roda, A., \& Daunert, S. (2002). Bioluminescence inmunoassay for cortisol using recombinant aequorin as a label. Analytical Biochemistry, 306(2), 204-211.

Montero, I., \& León, O. (2007). A guide for naming research studies in Psychology. International Journal of Clinical and Health Psychology, $7(3), 847-862$.

Moya-Albiol, L., Serrano, M. A., González-Bono, E., Rodríguez-Alarcón, G., \& Salvador, A. (2005). Respuesta psicofisiológica de estrés en una jornada laboral. Psicothema, 17(2), 205-211.

Murphy, M., Mazzocco, M., Gerner, G., \& Henry, A. (2006). Mathematics learning disability in girls with Turner Syndrome or fragile X Syndrome. Brain and Cognition, 61(2), 195-210.

Onder, A., Aycan, Z., Cetinkaya, S., Kendirci, H. N., Bas, V. N., \& Agladioglu, S. Y. (2012). Assessment of the 21-hydroxylase deficiency and the adrenal functions in young females with Turner Syndrome. Journal of Pediatric Endocrinology \& Metabolism, 25(7-8), 681-685.

Plomin, R., De Fries, J., McClearn, G., \& Guffin, P. (2002). Genética de la conducta. Buenos Aires, Argentina: Ariel Ciencia.

Ramos, F. (2003). Síndrome de Turner: manifestaciones clínicas. Sociedad Española de Endocrinología Pediátrica, 9, 1-12.

Ross, J., Roeltgen, D., \& Zinn, A. (2006). Cognition and the sex chromosomes: Studies in Turner Syndrome. Hormona Researchs, 65, 47-56.

Schmidt, P., Rubinow, D., \& Bondy, C. (2006). Adult women with Turner Syndrome: A systematic evaluation of current and past psychiatric illness, social functioning, and self-esteem. International Congress Series, 1298, 100-107.

Sutton, E. J., McInerney-Leo, A., Bondy, C. A., Gollust, S. E., King, D., \& Biesecker, B. (2005). Turner Syndrome: Four challenges across the lifespan. American Journal of Medical Genetics 139(A), 57-66.

Suzigan, L., Silva, R., Marini, S., Baptista, M., Guerra J., Magna, L., \& Maciel-Guerra, A. (2004). A percepção da doença em portadoras da Síndrome de Turner. Jornal de Pediatria, 80(4), 309-314.

Tamm, L., Menon, V., Allan L., \& Reiss, A. (2003). Abnormal prefrontal cortex function during response inhibition in Turner Syndrome: Functional magnetic resonance imaging evidence. Society of Biological Psychiatry, 53(2), 107-111.

Zabaletta, V., Gasparri, G., Gorostegui, M., Aguilar, M. J., \& López, M. (2011). Factores protectores y de riesgo en el estrés escolar. Implicancias en el Síndrome de Turner. Revista Latinoamericana de Ciencia Psicológica, 3(1), 30-37.

Zinn, A., Roeltgen, D., Stefanatos, G., Ramos, P., Elder, F., Kushner, H., ... Ross, J. (2007). A Turner Syndrome neurocognitive phenotype maps to Xp22.3. Behavioral and Brain Functions, 3(24), $1-14$. 